\title{
On the Streamwise and Normal Vortices Forming on Plates with Spanwise Periodic Cambering
}

\author{
Redha Wahidi ${ }^{1}$, James P. Hubner ${ }^{2}$ and Semih M. Ölçmen ${ }^{2}$ \\ ${ }^{1}$ Innovative and Renewable Energy Program, Kuwait Institute for Scientific Research, State of Kuwait \\ rwahidi@hotmail.com \\ ${ }^{2}$ Department of Aerospace Engineering and Mechanics, The University of Alabama, Tuscaloosa, AL, USA
}

\begin{abstract}
Three-dimensional measurements of the flow field over rigid plates with periodic cambering in the spanwise direction were performed. Two plates of an aspect ratio of 5 with either 5 or 9 periodic cells were used, and measurements were performed at three angles of attack and at a Reynolds number of 28,000. Previous research showed that organized streamwise and normal vortices form in the valleys between the periodic cells, and that these vortices contribute to slight enhancement of lift and reduction in drag. In this investigation, the details of these vortices are examined and compared at three different flow fields including: 1) short laminar separation bubble, 2) long laminar separation bubble and 3) fully separated flow. The formation of these primary vortices is observed in all cases. This investigation also discusses the formation of these vortices to provide further insight to the flow physics of these vortices. The formation of these vortices is influenced by the interaction between the periodic cambering and the thickness and strength of the backflow region. This interaction becomes strongest when a long laminar separation bubble forms resulting in larger vortices in size and magnitude. The instantaneous flow field shows that the primary vortices form most of the time, while they move in the spanwise direction and breakdown in the streamwise direction at other instances of time. In addition to the primary vortices, smaller streamwise and normal vortices also form. Proper Orthogonal Decomposition results indicate that reconstructing the flow field with different number of modes isolates the primary vortices from the smaller scale vortices.
\end{abstract}

\section{INTRODUCTION}

Micro air vehicles (MAVs) operate in a Reynolds number range between 20,000 and 200,000 where the performance of high Reynolds number wings deteriorates due to flow separation. This challenge has encouraged researchers to explore unconventional wing designs to improve performance and maneuverability of MAVs. Generally, low-Reynolds-number airfoils favor thin, cambered shapes. For a membrane wing with a similar configuration as in this investigation (i.e., multiple cells and fixed flat leading-edge, it exhibits an increased lift curve slope, lift coefficients at the post-stall region and maximum aerodynamic efficiency compared to the rigid counterparts [Zhang et al. 2012, Timpe et al. 2013]. Both time-averaged and dynamic characteristics of membrane wings affect the flow. The changes occur due to passive shape adaptation: changes in both time-averaged and dynamic cambering and washout (geometric twist) with angle of attack, dynamic pressure, pre-tension, membrane properties and membrane geometry [Zhang et al. 2014]. Membrane wings with a free trailing-edge in a free stream flow create a time-averaged cambering [Timpe et al. 2013]. For wings with multiple cells, a time-averaged, spatially-periodic pattern of positive cambering and local wash-out (negative geometric twist) is created. Since improvements in the aerodynamic performance are found in time-averaged force measurements [Zhang et al. 2012], the time averaged shape of the flexible membrane wing is expected to produce improvements in performance. Wahidi et al. (2014) investigated the effects of periodic cambering on the surrounding flow field and related the improved performance to the complex vortical structures on the periodically-cambered plates. They showed that the periodic cambering creates spanwise variations in the backflow region, Reynolds stresses, and spanwise vorticity, and that these spanwise variations are induced by streamwise and normal vortices. They also discussed the relationship between these vortices and the enhancement of aerodynamic performance. The streamwise and normal vortices were shown to become more organized for a flow with a long laminar separation bubble when compared to those form when the flow is fully separated. The current investigation examines these vortices in more detail including the effect of angle of attack and the mechanisms influencing their formation. This detailed investigation of the streamwise and normal vortices provides a further insight of the interaction of the flow field with the time-averaged cambering. This insight is an important step toward a better understanding of the interaction of the flow field with flexible membrane wings with multiple cells. 
The aim of this investigation is to compare the streamwise and normal vortices at different angles of attack representing different flow fields with short and long laminar separation bubbles and a fully separated flow, and examine their details by means of the time-averaged and instantaneous flow fields, as well as the results of the Proper Orthogonal Decomposition (POD) analysis.

\section{EXPERIMENTAL FACILITY, TEST ARTICLES AND PROCEDURE}

The flow field measurements were conducted in a water tunnel facility with dimensions of 108 inches $(2.743 \mathrm{~m}), 15$ inches $(0.381 \mathrm{~m})$ and 30 inches $(0.762 \mathrm{~m})$ for the length, width and height, respectively. The tunnel's manufacturer reports a turbulence level of $0.4 \%$ at a freestream velocity of $2 \mathrm{in} / \mathrm{sec}(0.051 \mathrm{~m} / \mathrm{s})$.

The two test articles (Fig. 1) are plates with periodic cambering of 9 cells $(9 \mathrm{CP})$ and 5 cells $(5 \mathrm{CP})$. The design of the cambered plate was inspired by an over-simplified model of a static or time-averaged membrane wings [Ifju et al 2006, Tamai et al 2008, Hubner and Hicks 2011] under aerodynamic load. The plates have an aspect ratio of 5 where the chord length and span are 3 inches $(7.62 \mathrm{~cm})$ and 15 inches $(38.1 \mathrm{~cm})$, respectively. The cambered shape was simplified as a circular arc at the trailing-edge with an arc height of $3.2 \mathrm{~mm}$ (or $4 \%$ relative to the chord) rising above the flat portion of the upper surface of the plate. This is a nominal value based on typical free stream conditions and membrane properties. The spanwise arc was then extruded with decreasing height until it reached the leading edge of the cell. The aspect ratios of the cells (cell span-to-cell chord) are 0.5 and 1.0 for the $9 \mathrm{CP}$ and 5CP, respectively. Each cell extends over the latter $80 \%$ of the plate in the chordwise direction. At the wing tips, the plate is flat and the spanwise gap between the cells is $3.8 \mathrm{~mm}(0.15$ in). The periodic pattern creates both geometric (negative, the TE is elevated relative to the LE) and aerodynamic (positive, the camber is concave down) twist of the wing

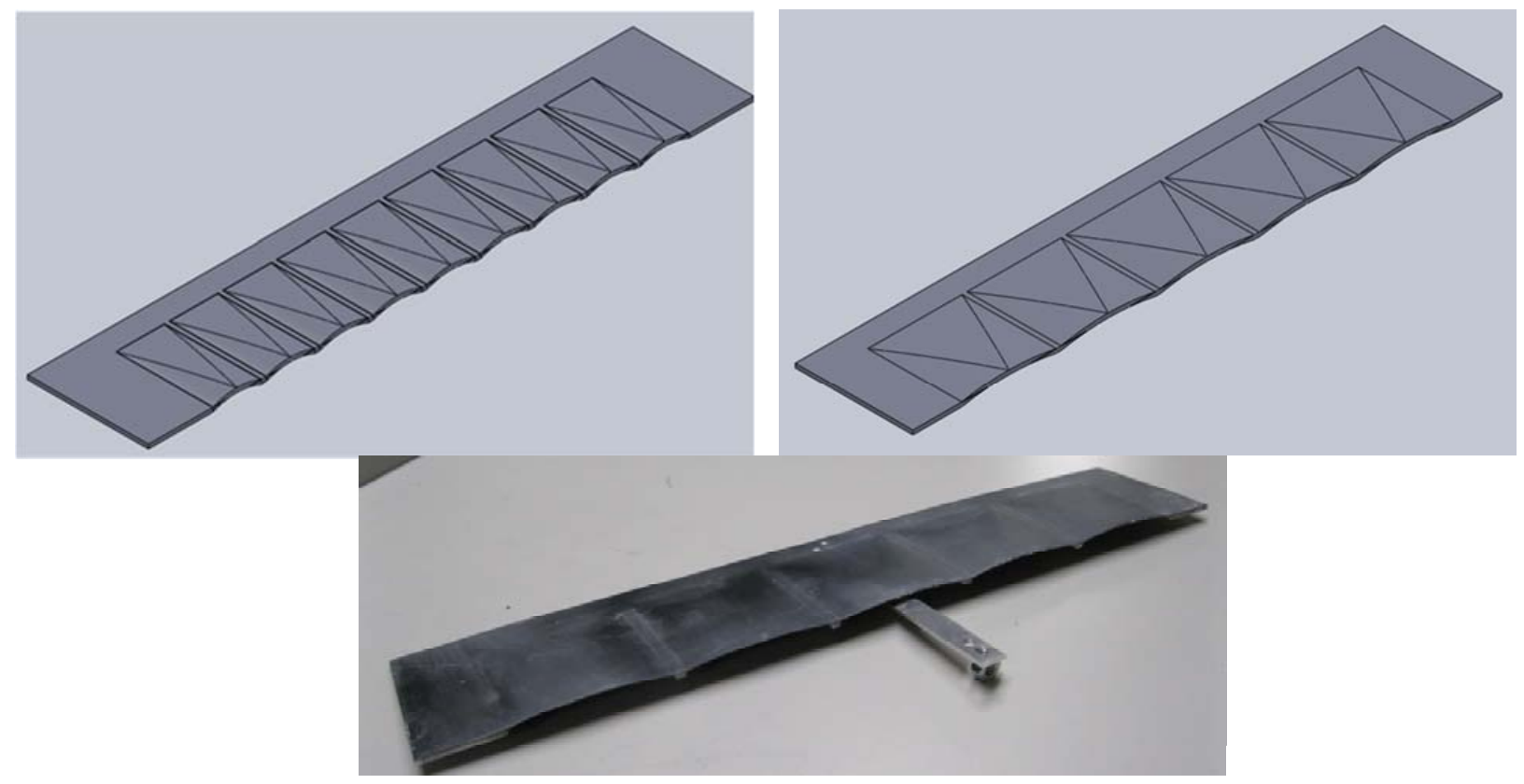

Figure 1. Oblique View (SolidWorks Model): 1:2 cell aspect ratio 9CP (left), 1:1 cell aspect ratio 5CP (right) and a photo of $5 \mathrm{CP}$ (bottom)

The flow field measurements were conducted at a Reynolds number based on the chord and free stream velocity $\left(R e_{c}\right)$ of 28,000 at angles of attack of $4^{\circ}, 8^{\circ}$ and $12^{\circ}$.

The flow field around the plates was measured using a volumetric three-component velocimetry (V3V). The flow was seeded with $50 \mu \mathrm{m}$ polymer particles, and the measurement volume was illuminated with a $425 \mathrm{~mJ} / \mathrm{pulse}$, dual-cavity $\mathrm{Nd}$ :YAG lasers. The laser pulses were spread into laser volumes and directed into the water tunnel through the glass bottom 
of the tunnel. The boundaries of the illumination volume were further set by masking the region away from the test articles to concentrate the illumination to the region of interest and to increase the vector concentration. The plates were vertically placed in the water tunnel using an attachment at the trailing edge of the lower surface of the plate, and the camera faces the top surface of the plate. A typical image yields 12,000 independent randomly-spaced velocity vectors that are interpolated onto a rectangular grid covering the measurement volume of $120 \mathrm{~mm}$ by $120 \mathrm{~mm}$ by $100 \mathrm{~mm}$ in the streamwise, spanwise and normal directions, respectively. The grid nodes are spaced at $3.15 \mathrm{~mm}$. The streamwise distance of the measurement volume covers the entire chord of the plate, whereas the spanwise distance covers 2 and 4 valleys between the cells for the $5 \mathrm{CP}$ and 9CP, respectively. The uncertainty in the velocity measurements is estimated to be $1 \%$ in the $x$ and $y$ directions $(u$ and $v$ velocity components) and $4 \%$ in the $z$ direction ( $w$ velocity component). A total of 250 statistically-independent instantaneous realizations (images) are captured at $3.6 \mathrm{~Hz}$.The streamwise, spanwise and vertical coordinates (tunnel coordinates) used in this investigation are $x, y$ and $z$, respectively, and their corresponding velocity components are $u, v$ and $w$ (see Wahidi et al. 2014 for more details on the plates, experimental setup and flow field measurement technique).

\section{PROPER ORTHOGONAL DECOMPOSITION FORMULATION}

Proper Orthogonal Decomposition (POD) analysis is a useful tool to analyze complex flow fields and identify dominant structures and events in the flow. It was first introduced as a tool for turbulent flow analysis [Lumley 1967] and has been extensively used to analyze computational and experimental data. In performing POD analysis, the coherence of the fluctuations in a flow field is identified by considering the energy contained in the set of modes generated by the POD analysis. Modes with the largest energy content (largest eigenvalues) are associated with dominant structures, and they provide an average spatial description of the structures [Kostas et al. 2005]. Also, when vortical structures of different scales and generated by different mechanisms are present in the flow, they are expected to have different energy content in the POD analysis. Reconstructing the flow using individual modes can isolate the different structures and provide valuable information on their dominance and details. The ability to isolate the structures requires that different structures in the flow to be coherent and have different frequency range.

The snapshot POD analysis (Sirovich 1987) is applied to the fluctuating velocity fields. Each of the 250 statisticallyindependent realizations is considered a snapshot, and the analysis is performed following the procedure described herein. The first step is to calculate the mean at each grid point in the measurement domain for each of the three-components of the velocity vectors using Eq. 1. The fluctuating velocity is calculated using Eq. 2.

$$
\begin{gathered}
\overline{\boldsymbol{U}}(\boldsymbol{X})=\frac{1}{N} \sum_{n=1}^{N} \boldsymbol{U}\left(\boldsymbol{X}, t_{n}\right) \\
\boldsymbol{U}^{\prime}\left(\boldsymbol{X}, t_{n}\right)=\boldsymbol{U}\left(\boldsymbol{X}, t_{n}\right)-\overline{\boldsymbol{U}}(\boldsymbol{X}),
\end{gathered}
$$

where $\boldsymbol{U}=(u, v, w), \boldsymbol{X}=(x, y, z)$, subscript index ( $n)$ runs through $N$ number of snapshots (i.e., 250 images), and superscript index $(m)$ represent the grid locations. The matrix containing the field variables is arranged as per Eq. 3.

$$
\boldsymbol{U}=\left[\begin{array}{ccccccccc}
\boldsymbol{u}_{1}^{1} & \ldots & \boldsymbol{u}_{1}^{\boldsymbol{M}} & \boldsymbol{v}_{1}^{1} & \ldots & \boldsymbol{v}_{1}^{\boldsymbol{M}} & \boldsymbol{w}_{1}^{1} & \ldots & \boldsymbol{w}_{1}^{\boldsymbol{M}} \\
\boldsymbol{u}_{2}^{1} & \ldots & \boldsymbol{u}_{2}^{\boldsymbol{M}} & \boldsymbol{v}_{2}^{1} & \ldots & \boldsymbol{v}_{2}^{\boldsymbol{M}} & \boldsymbol{w}_{2}^{1} & \ldots & \boldsymbol{w}_{2}^{\boldsymbol{M}} \\
\vdots & \vdots & \vdots & \vdots & \vdots & \vdots & \vdots & \vdots & \vdots \\
\boldsymbol{u}_{\boldsymbol{N}-1}^{1} & \cdots & \boldsymbol{u}_{\boldsymbol{N}-1}^{\boldsymbol{M}} & \boldsymbol{v}_{\boldsymbol{N}-1}^{1} & \ldots & \boldsymbol{v}_{\boldsymbol{N}-1}^{\boldsymbol{M}} & \boldsymbol{w}_{\boldsymbol{N}-1}^{1} & \cdots & \boldsymbol{w}_{\boldsymbol{N}-1}^{\boldsymbol{M}} \\
\boldsymbol{u}_{\boldsymbol{N}}^{1} & \cdots & \boldsymbol{u}_{\boldsymbol{N}}^{\boldsymbol{M}} & \boldsymbol{v}_{\boldsymbol{N}}^{1} & \ldots & \boldsymbol{v}_{\boldsymbol{N}}^{\boldsymbol{M}} & \boldsymbol{w}_{\boldsymbol{N}}^{1} & \ldots & \boldsymbol{w}_{\boldsymbol{N}}^{\boldsymbol{M}}
\end{array}\right]
$$

The time correlation tensor, $\boldsymbol{R}$, for the velocity field is determined as per Eq. 4 , and it returns an $N$ by $N$ matrix.

$$
\boldsymbol{R}=\boldsymbol{U}^{\prime} \boldsymbol{U}^{\prime \boldsymbol{T}},
$$


The eigenfunction problem stated in Eq. (5) was solved using an in-house written Matlab® code

$$
R A^{i}=\lambda^{i} A^{i}
$$

where $A^{\mathrm{i}}$ are the temporal eigenfunctions and $\lambda^{i}$ are the eigenvalues. The eigenvalues represent the energy within each mode and the sum of the eigenvalues represent the total energy $(E)$ contained in the flow field. This "energy" represents the kinetic energy density, $\boldsymbol{K} \boldsymbol{E}=\frac{1}{2} \rho(\boldsymbol{U}, \boldsymbol{U})=\frac{1}{2} \rho\|\boldsymbol{U}\|^{2}$. The POD modes (spatial eigenfunctions $\boldsymbol{\phi}^{\text {i) }}$ were then calculated as per Eq. 6 and normalized to have orthonormal basis.

$$
\phi^{i}=\frac{\sum_{n=1}^{N} A_{n}^{i} U^{\prime n}}{\left\|\sum_{n=1}^{N} A_{n}^{i} U^{\prime n}\right\|}
$$

The Euclidean norm appearing in Eq. 6 is defined as: $\|\boldsymbol{Y}\|=\sqrt{\mathbf{Y}_{1}^{2}, \boldsymbol{Y}_{2}^{2}, \ldots, \boldsymbol{Y}_{\boldsymbol{N}}^{2}}$, where $\boldsymbol{Y}$ is dummy variable representing $A U^{\prime}$. The flow field can be reconstructed by first calculating the POD coefficients (temporal coefficients) according to Eq. 7 and then using Eq.8 with a specific mode and/or a specific number of modes. The POD coefficients represent a projection of the velocity field onto the spatial eigenfunctions. The vorticity spatial modes are calculated by taking the curl of the velocity spatial modes (results of Eq. 6).

$$
\begin{aligned}
& \boldsymbol{a}_{\boldsymbol{i}}=\boldsymbol{\phi}^{\boldsymbol{i}} \boldsymbol{U}^{\mathbf{n}^{\boldsymbol{n}}} \\
& \boldsymbol{u}^{\boldsymbol{n}}=\sum_{\boldsymbol{i}=1}^{N} \boldsymbol{a}_{i}^{n} \boldsymbol{\phi}^{i}
\end{aligned}
$$

The POD analysis is applied to 2D planes using the three components of the velocity vector. It has been shown (Wahidi et al. 2015) that applying the POD analysis to the entire 3D3C measurement domain produces spatial modes that capture the vortical structures in the flow; however, when the POD analysis is performed using the three components of the velocity vector in a 2D plane, the spatial distribution of POD modes become visually clearer.

\section{RESULTS}

\subsection{Flow Field Results}

The three angles of attack create three distinct flow fields. At $4^{\circ}$ and $8^{\circ}$ the separated shear layer reattaches, creating a short laminar separation bubble at $4^{\circ}$ and a long laminar separation bubble at $8^{\circ}$. The separation bubble at $8^{\circ}$ is characterized by a reverse-flow region covering the majority of the chord at the centerline, and a relatively high separated shear layer. A fully separated flow with no reattachment of the separated shear layer is encountered at $12^{\circ}$.

The streamwise and normal vorticity components are shown in $y-z$ planes extending from $x / c=0.25$ to 1.0 in $0.25 \mathrm{c}$ increments in both figures. Additionally, the normal vorticity is also shown in an $x-y$ plane at a $z / c$ location above the backflow region. These planes are shown in the schematic in Fig. 2. The streamwise and normal components of the vorticity at different angles of attack are shown in Figs. 3 and 4, respectively. Also included in the planes iso-lines defined by the vorticity and colored by $\lambda_{2}$ values (Jeong and Hussain 1995) to distinguish between the vorticity that is associated with vortices and that is developing due to shear or velocity gradient.

The vortices show distinct structures at different angles of attack, but universal trends are also observed. In general, streamwise vortices of opposite directions form on each side of the plate centerline, and they extend from $x / c=0.25$ to 1.0. These vortices form in the valleys between the cells, and the cell dependency is also obvious. 


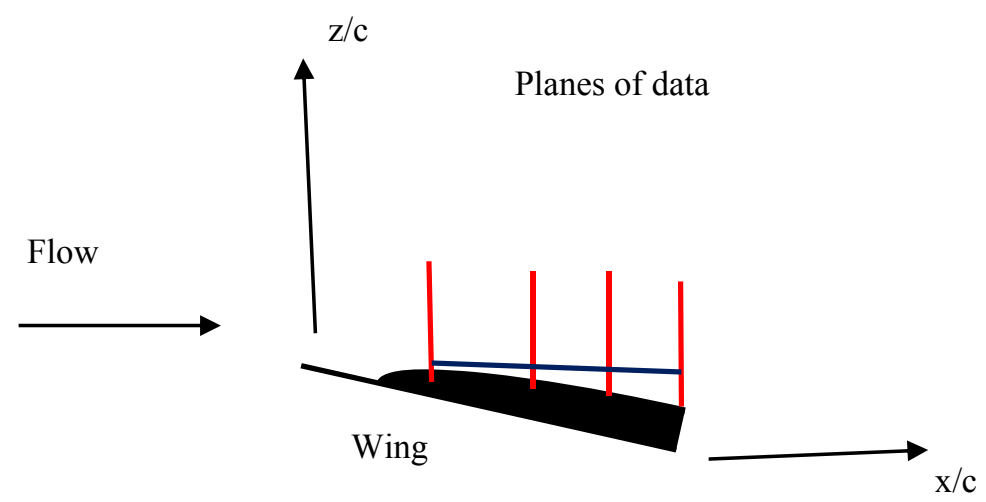

Figure 2. Schematic of the planes where the data is shown in subsequent figure

At $4^{\circ}$, a short laminar separation bubble forms where the mean reattachment occurs at $x / c=0.3$ and the separated shear layer maximum height is approximately $0.1 c$ above the plate. In this case, the streamwise vortices are largest in size and magnitude at $x / c=0.3$ before their size and magnitude decrease further downstream. When a long separation bubble forms at $8^{\circ}$, with a mean reattachment at $x / c=0.95$ and a separated shear layer maximum height of about $0.15 c$, the vortices become more organized and with an increase in magnitude and size. In contrast to the $4^{\circ}$ case, the streamwise vortices at $8^{\circ}$ grow in the downstream direction. The vortices in the fully separated flow case at $12^{\circ}$ are less organized. However, similar to the case at $8^{\circ}$, their magnitude grows in the streamwise direction. The largest streamwise vortices exist at approximately $x / c=0.3,0.75$ and 1.0 for $4^{\circ}, 8^{\circ}$ and $12^{\circ}$ cases, respectively.

The normal vortices are connected to the streamwise vortices creating hairpin-like structures. The connection is recognized by the correspondence of the normal vortices in the $x-y$ plane with the streamwise vortices in the $y-z$ planes (compare Figs. 3 and 4). The normal vortices at $4^{\circ}$ exhibit larger vortices (referred to as primary vortices herein) of opposite sign about the midspan location. Also, smaller structures beginning at the leading edge are observed in the $4^{\circ}$ case. At $8^{\circ}$, the primary vortices become more organized and smaller-scale vortices with opposite sign (referred to as secondary vortices herein) form between the primary vortices. The organization of these vortices deteriorates at $12^{\circ}$. In general, however, the structures in the $9 \mathrm{CP}$ case are visually clearer.

As mentioned above, the primary vortices on each half of the plate with respect to the centerline have the same sign, as illustrated in Fig. 5. The figure also illustrates the connection between the streamwise and normal vortices and the secondary vortices of opposite sign between the primary vortices. 


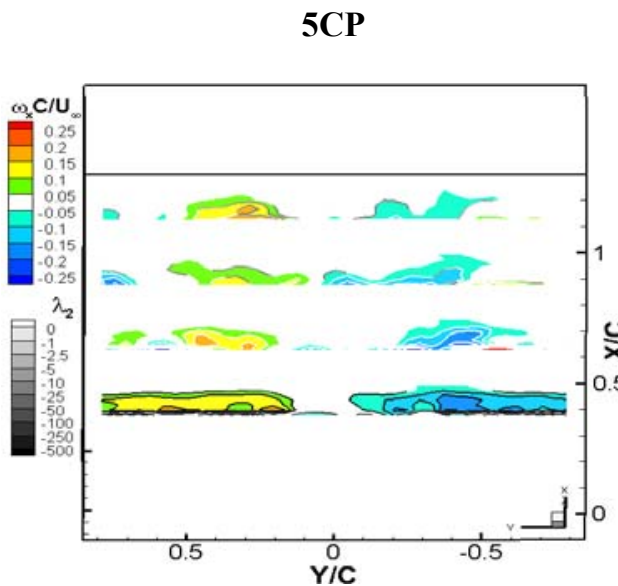

(a)

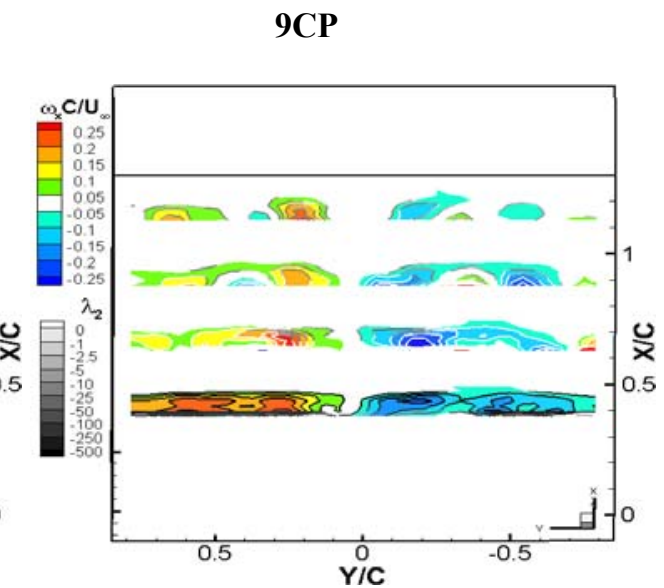

(b)

$4^{\circ}$

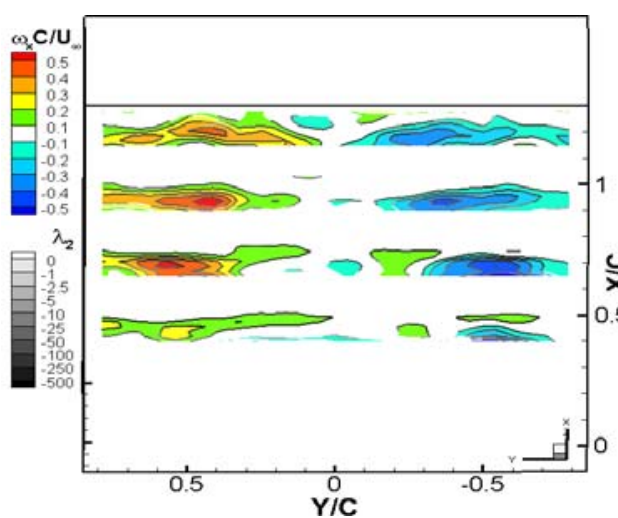

(c)

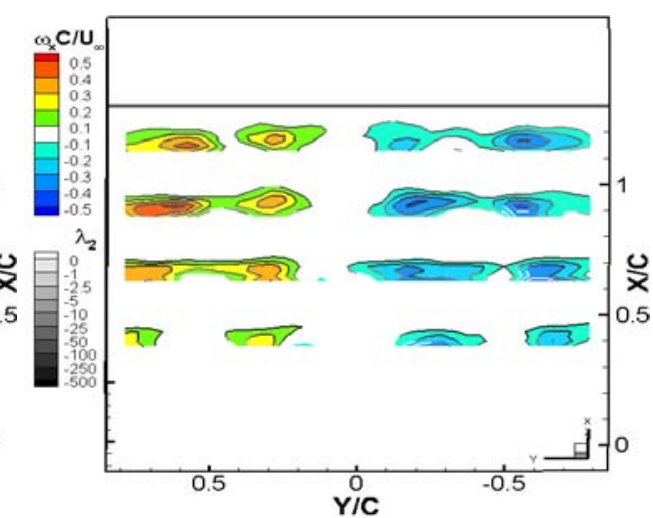

(d)

$8^{\circ}$

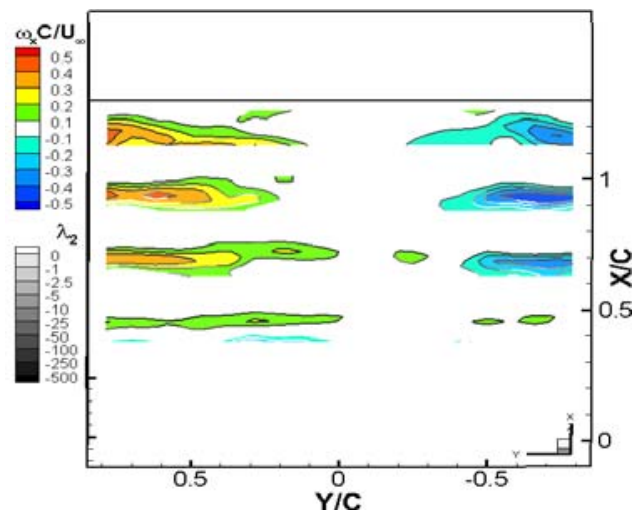

(e)

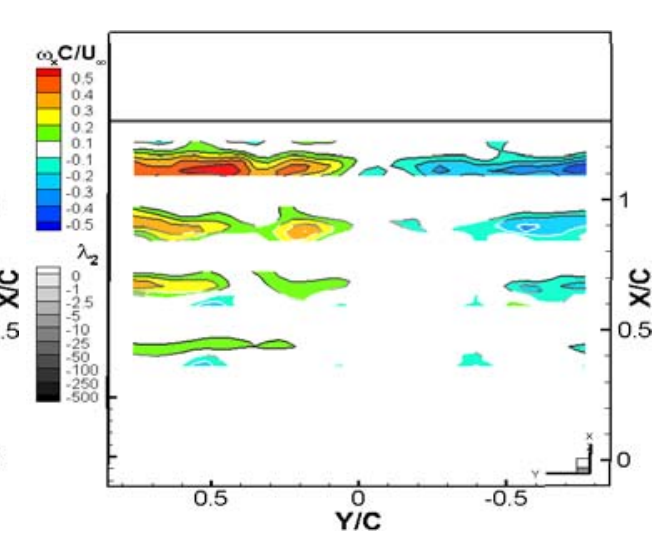

(f)

$12^{\circ}$

Figure 3. Streamwise vorticity $\left(\omega_{\boldsymbol{X}}\right)$ presented on $y$-z planes at $x / c=0.25$ to $1.0 \mathrm{in} 0.25 \mathrm{c}$ increments. Iso-lines defined by $\left(\omega_{x}\right)$ and colored by $\lambda_{2}$ values; Left: 5CP, Right: $9 \mathrm{CP}$; (a,b) $4^{\circ},(\mathrm{c}, \mathrm{d}) 8^{\circ},(\mathrm{e}, \mathrm{f}) 12^{\circ}$ 


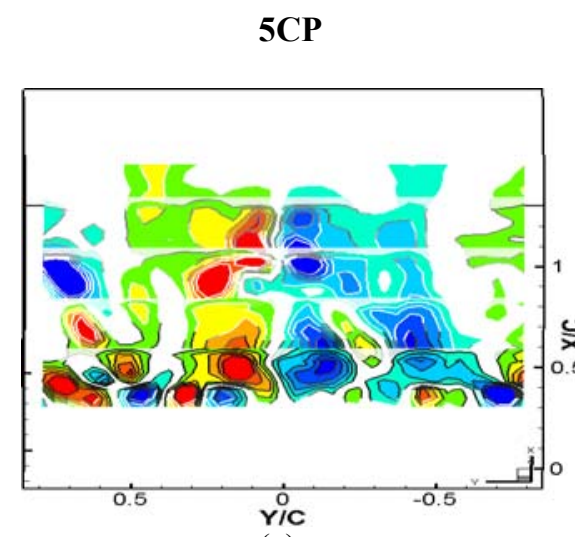

(a)

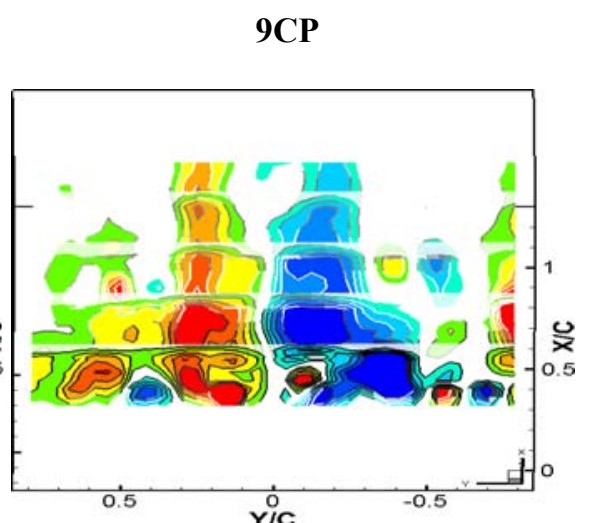

(b)

$4^{\circ}$

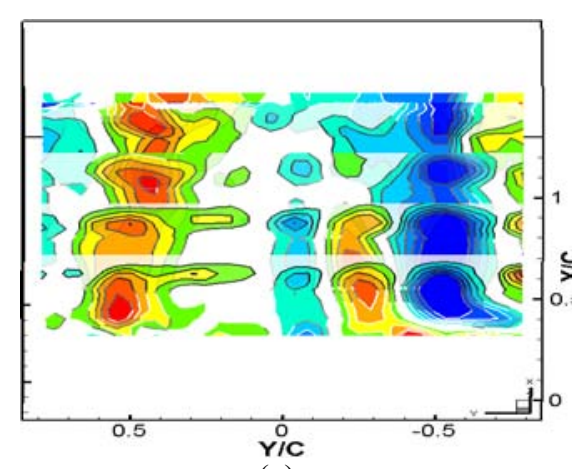

(c)

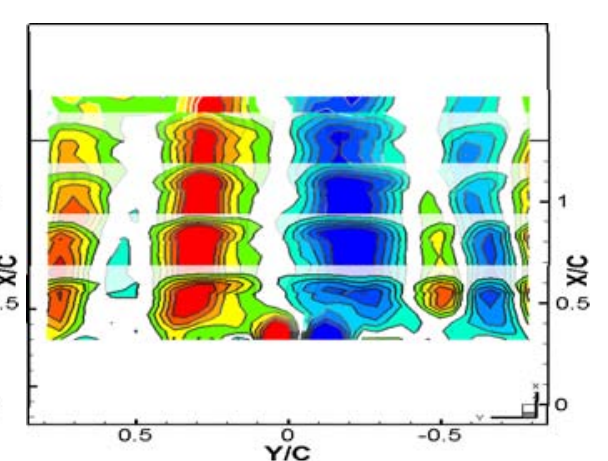

(d)

$8^{\circ}$

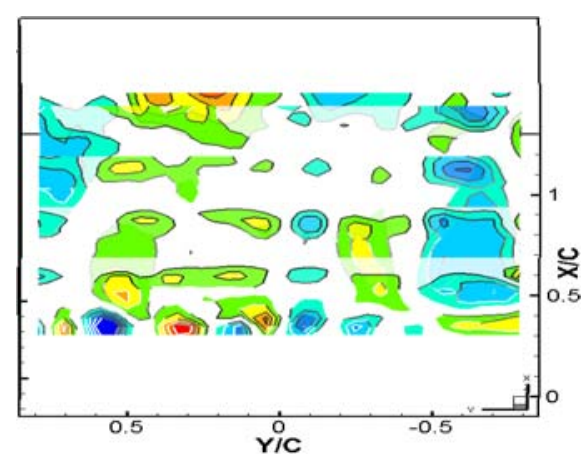

(e)

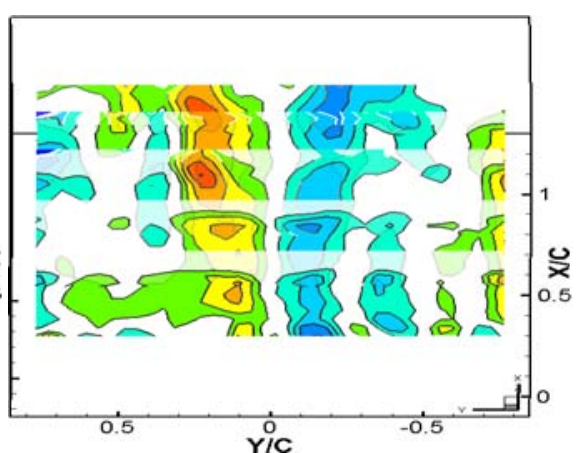

(f)

$12^{\circ}$

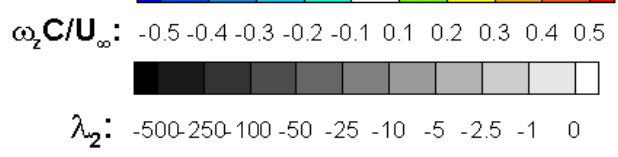

Figure 4. Normal vorticity $\left(\omega_{z}\right)$ presented on $y$-z planes at $x / c=0.25$ to 1.0 in $0.25 \mathrm{c}$ increments and on a $x-y$ plane. Isolines defined by $\left(\omega_{z}\right)$ and colored by $\lambda_{2}$ values; Left: $5 \mathrm{CP}$, Right: $9 \mathrm{CP}$; (a,b) $4^{\circ},(\mathrm{c}, \mathrm{d}) 8^{\circ}$, (e,f) $12^{\circ}$ 


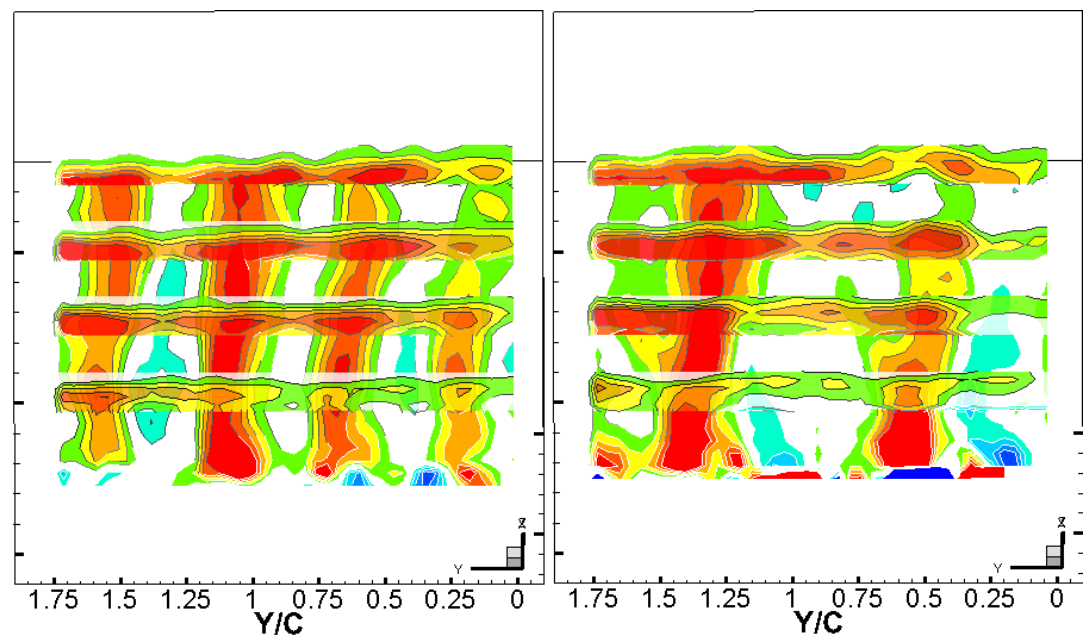

(a)

(b)

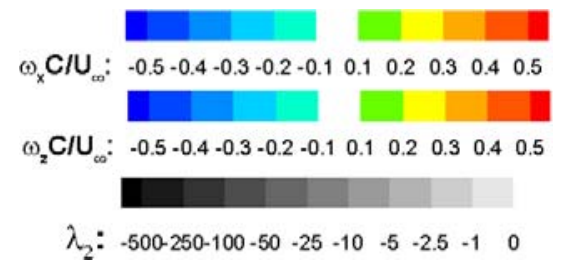

Figure 5. Normal vorticity $\left(\omega_{z}\right)$ presented on $x-y$ plane and streamwise vorticity $\left(\omega_{x}\right)$ on multiple $y-z$ planes at $x / c=0.25$ to 1.0 in $0.25 \mathrm{c}$ increments. Iso-lines defined by the corresponding vorticity and colored by $\lambda_{2}$ values. (a) $9 \mathrm{CP}$ at $8^{\circ}$, (b) $5 \mathrm{CP}$ at $8^{\circ}$

The streamwise and normal vortices are predicted to be present at all instants of time similar to a tip vortex forming on a flat plate at an angle of attack. However, the streamwise and normal vortices are quasi-steady since they form and breakdown as well as meander in the spanwise direction. Figures $6 \mathrm{a}$ and $6 \mathrm{~b}$ are compared to Figs. $3 \mathrm{c}$ and $4 \mathrm{c}$, respectively, to illustrate that the vortices centered at $y / c=-0.5$ and 0.5 are visible (see arrows in Fig. 6b). However, at another instant of time (Fig. 6c-d) these vortices move in the spanwise direction (see arrows in Fig. 6c) and breakdown in the streamwise direction (see arrow in Fig. 6d). In addition to the primary vortices, smaller counter rotating vortices also appear in the instantaneous snapshots that do not appear in a time-averaged sense. Some of these vortices form on the cambered cells away from the ridges. The reason for the formation of some of these vortices is the concave shape of the cells in the spanwise direction. These vortices are not similar to the streamwise and normal counter rotating vortices found in the reattachment region on an airfoil (Wahidi et al. 2013) since they form prior to the reattachment of the separated shear layer. 


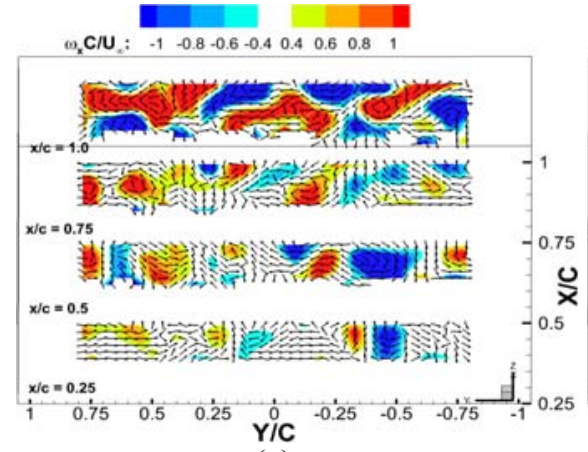

(a)

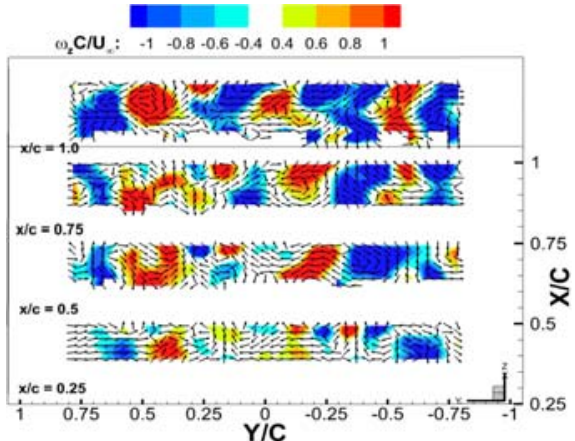

(b)

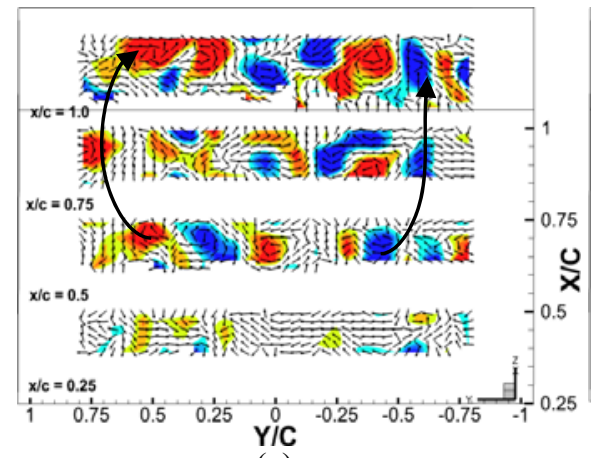

(c)

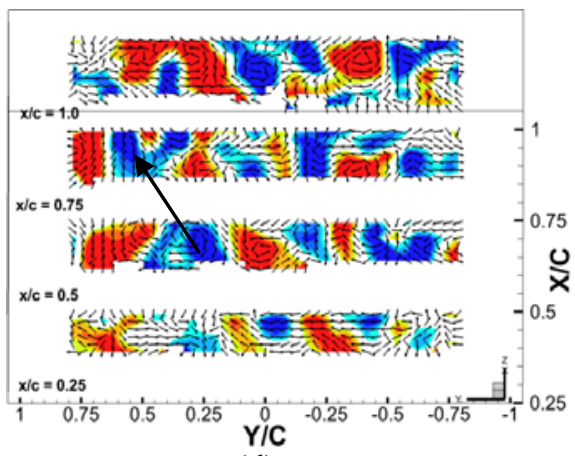

(d)

Figure 6. Instantaneous snapshots of $5 \mathrm{CP}$ at $8^{\circ}$ showing $y$ - $z$ planes colored by $(\mathrm{a}, \mathrm{c})$ streamwise vorticity $\left(\omega_{\boldsymbol{x}} \boldsymbol{C} / \boldsymbol{U}_{\infty}\right)$ and $(\mathrm{b}, \mathrm{d})$ normal vorticity $\left(\omega_{z} C / U_{\infty}\right)$

\section{$\underline{4.2 \text { POD Results }}$}

The POD analysis is performed at 2D $y$-z planes referred to as Plane 1 , Plane 2 and Plane 3 located at $x / c=0.25,0.5$ and 0.75 , respectively. As mentioned above, the POD analysis is performed on the velocity vectors (i.e., $u, v$ and $w$ ), and the curl of the resulting POD modes is taken to represent vorticity vector POD modes.

The eigenvalues of the POD analysis of the 5CP and 9CP plates at different angles of attack are shown in Fig. 7. At Plane 1, eigenvalues for all the cases are close to each other making difficult to extract any conclusions from the POD analysis. This indicates that the variations of the structures in the flow at different angles of attack begin further downstream. However, although the energy at Plane 1 gradually decreases, it does not represent the energy distribution of a turbulent flow where the energy is distributed over a wider range of modes due to the presence of small scale structures. 


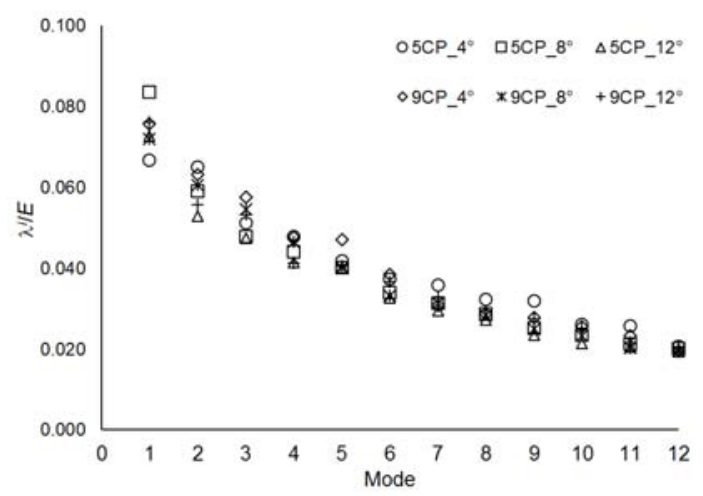

(a)

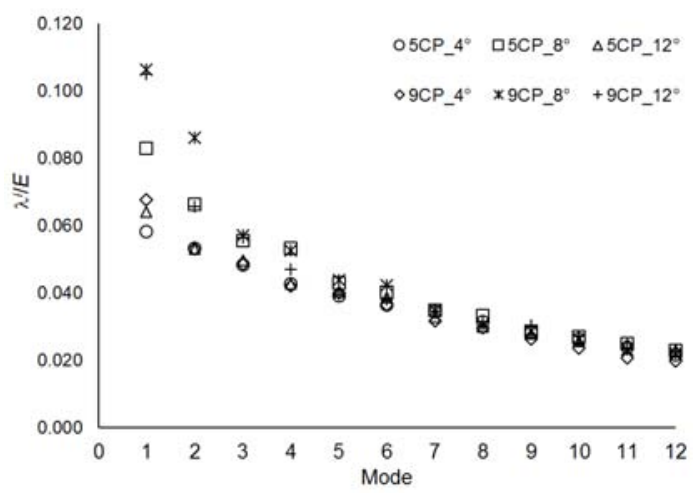

(b)

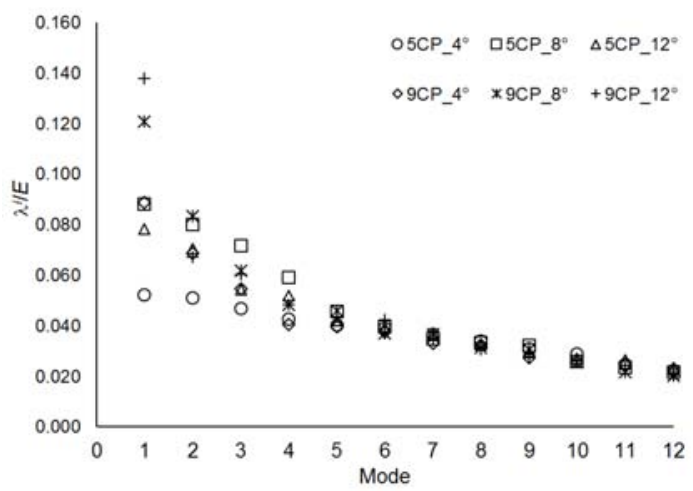

(c)

Figure 7. Energy fraction $\left(\lambda^{i} / E\right)$ of the first 12 modes, (a) Plane 1, (b) Plane 2, (c) Plane 3

At Plane 2, mode 1 for the cases at $8^{\circ}$ and $12^{\circ}$ is more dominant. The primary vortices that are captured in this mode increase in size and magnitude at these angles of attack causing higher energy content.. The first four modes at $8^{\circ}$ contain higher energy than the ones for the $12^{\circ}$ case which is higher than the energy content of these modes at $4^{\circ}$. The higher energy contained in the first 4 modes at $8^{\circ}$ reflects the higher magnitude and size of the streamwise and normal vortices. Moreover, the first two modes of the $9 \mathrm{CP}$ contain larger energy than those of the $5 \mathrm{CP}$ which could be due to the twice as many primary vortices captured in spanwise direction. This is also observed at $4^{\circ}$ and $12^{\circ}$, but in these cases the energy content of the $9 \mathrm{CP}$ is only slightly higher. At Plane 3 , the energy distribution at $4^{\circ}$ does not considerably change, whereas at 
$12^{\circ}$ it has higher values in the first 4 modes. The energy content of the first 4 modes at $8^{\circ}$ remains the largest, but unlike at Plane 2, the 5CP plate has higher values. The energy content is likely to increase due to the larger vortices in the 5CP at this plane (compare Fig. $3 \mathrm{c}$ and $3 \mathrm{~d}$ ).

When the mean is not subtracted from the velocity field, the first POD mode approximately represents the timeaveraged results. This mode is referred to here as mode zero, and it only shows the primary vortices (Fig 8).

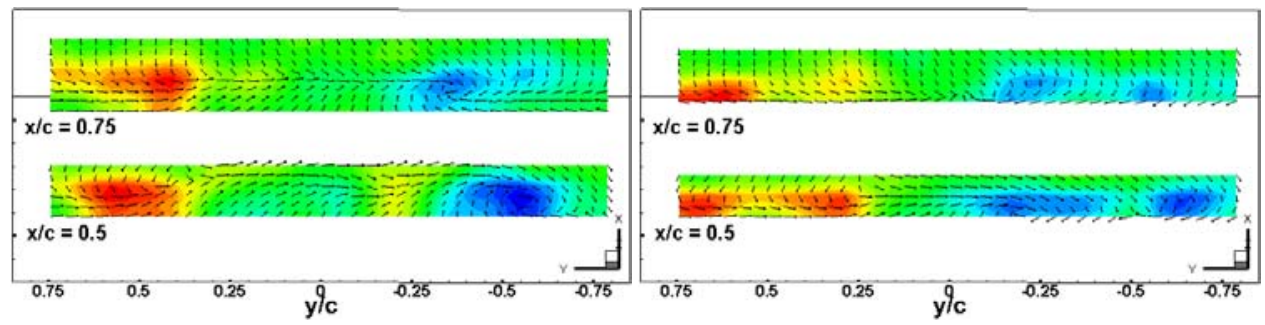

(a)

(b)

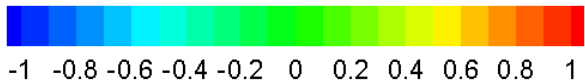

Figure 8. POD mode 0 of $\phi_{\omega_{X}}^{0}$ at Planes 2-3 (x/c =0.5 and 0.75$)$ with vectors defined by $\phi_{U}^{0}$. (a) $5 \mathrm{CP}$ at $8^{\circ}$, (b) $9 \mathrm{CP}$ at $8^{\circ}$

As mentioned in the flow field results, primary vortices form between the cells, as well as smaller vortices. If the mechanisms influencing the formation of these vortices are different, it is possible to isolate the primary and smaller vortices using the POD modes. In order to evaluate this possibility, and in order to explain the dominance of mode 1 in Planes 2 and 3 at $8^{\circ}$ and $12^{\circ}$, the spatial distributions of POD modes 1 and 2 are examined. Figure 9 shows the first POD mode of the streamwise vorticity ( $\phi_{\omega_{X}}^{1}$ ) in Planes 1-3 for the 5CP and 9CP at different angles of attack. Regions of large magnitudes in Planes 2 and 3 of $9 \mathrm{CP}$ at $8^{\circ}$ (Fig. 9d) correspond to the primary vortices. The primary vortices centered at $y / c$ $= \pm 0.25$ and 0.5 (Fig. $3 \mathrm{~d}$ ) are combined in one region with larger magnitudes at $y / c= \pm 0.25$. At Plane 1 of this case, traces of the structures appearing in Planes 2 and 3 are observed but with less obvious circular shapes. This is due to the smaller size and magnitude of the vortices at this plane (compare Plane 1 of Figs. 9d and 3d). The spatial distributions in Planes 2 and 3 of $5 \mathrm{CP}$ at $8^{\circ}$ (Fig. 9c) have a structure at $y / c=+0.35$ as well as a structure with opposite sign at midspan. The structures at $y / c=0$ and -0.35 correspond to the primary vortices but their position is shifted in the spanwise direction (compare Planes 2-3 of Figs. 9c and 3c). It appears that the primary vortices are well captured at Planes 2 and 3 of $9 \mathrm{CP}$ at $8^{\circ}$. This is reflected in the distribution of the energy content where $9 \mathrm{CP}$ at $8^{\circ}$ has larger values at mode 1 .

The spatial distributions in Planes 2 and 3 at $12^{\circ}$ show structures at $y / c=0.5$ and 0.35 for $5 \mathrm{CP}$ and $9 \mathrm{CP}$, respectively. These regions of large magnitudes are related to the primary vortices. The primary vortices on the other side of the midspan location are not captured because they have a smaller size and magnitude (Figs. 3e-f). The primary vortices in Plane 3 at $4^{\circ}$ are captured for both plates (Figs. 9a-b), whereas they are only captured in Plane 2 of 9CP (Fig. 9b).

At mode $2\left(\phi_{\omega_{X}}^{2}\right)$, the spatial distributions (Fig. 10) in all planes are different, which suggests that the structures are not related to primary vortices. Moreover, the difference between the spatial distributions of modes 1 and 2 indicate that they are not coupled as can be confirmed by the energy distributions (Fig. 7b-c) where the energy contents at these modes are different. At mode 2, the spatial distributions in Plane 2 of $9 \mathrm{CP}$ and $5 \mathrm{CP}$ at $8^{\circ}$ are similar.

The above results suggest that the primary vortices are captured in mode 1 , and that the similarity in the spatial distributions in both Planes 2 and 3 is related to their extension in the streamwise direction without movement in the spanwise direction nor a breakdown in the streamwise direction. The results also suggest that mode 2 onward capture the smaller vortices observed in the instantaneous snapshots (Fig. 6). These smaller vortices meander in the spanwise direction as well as breakdown in the streamwise direction. 


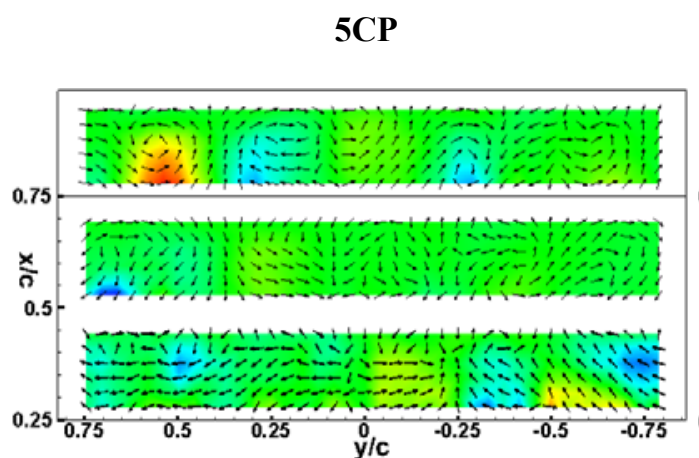

(a)

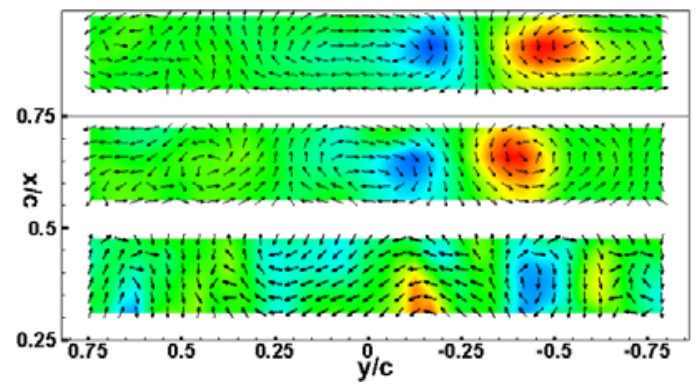

(c)

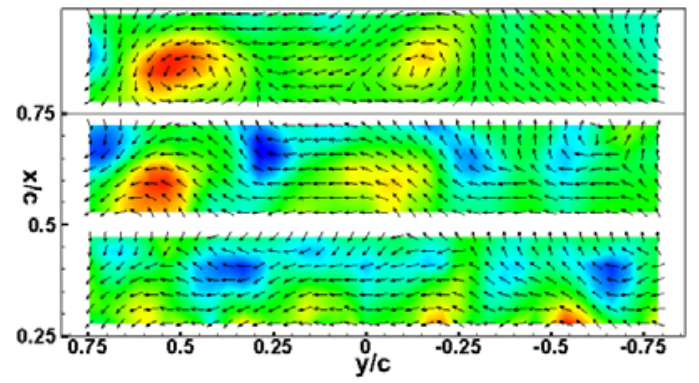

(e)

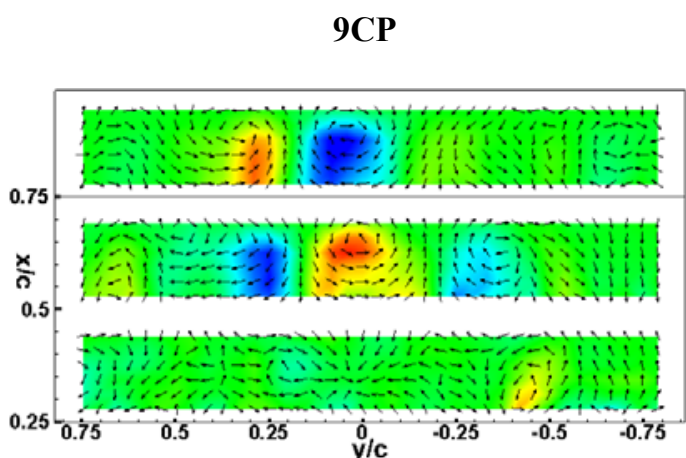

(b)

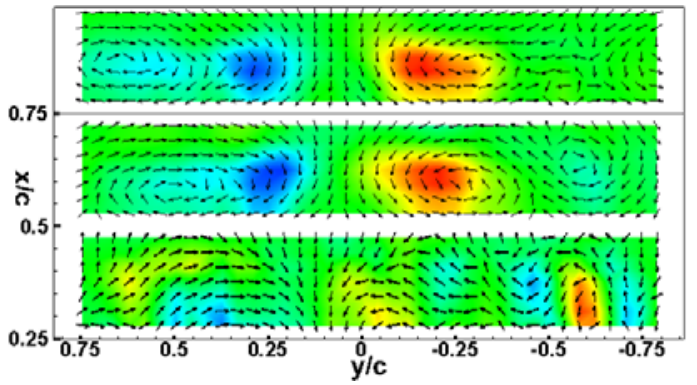

(d)

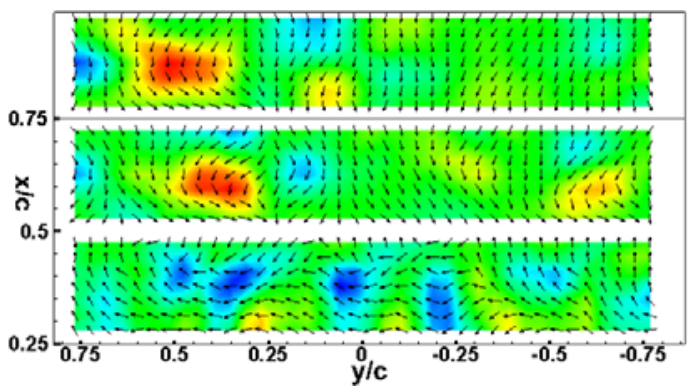

(f)

Figure 9. POD mode 1 of $\phi_{\omega_{X}}^{1}$ at Planes 1-3 $(x / c=0.25,0.5$ and 0.75$)$ with vectors defined by $\phi_{U}^{1}$. Left: $5 \mathrm{CP}$, Right: $9 \mathrm{CP}$ $(\mathrm{a}, \mathrm{b}) 4^{\circ},(\mathrm{c}, \mathrm{d}) 8^{\circ},(\mathrm{e}, \mathrm{f}) 12^{\circ}$ 


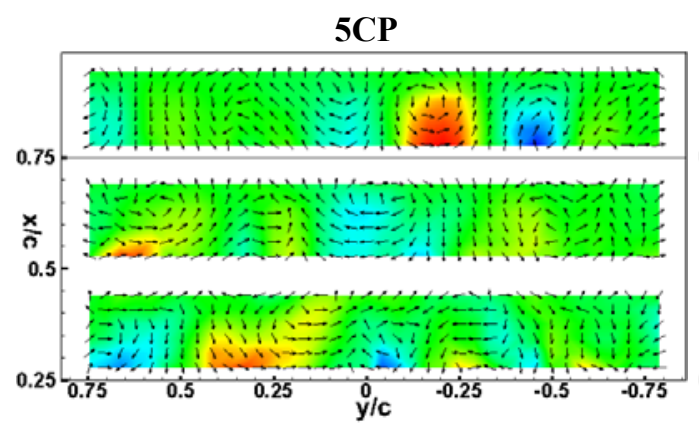

(a)

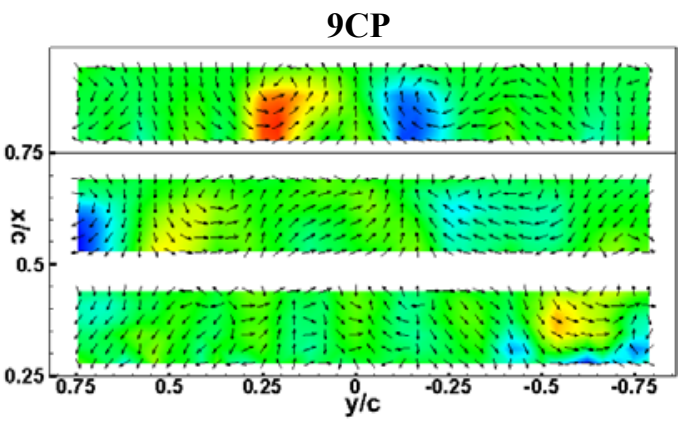

(b)

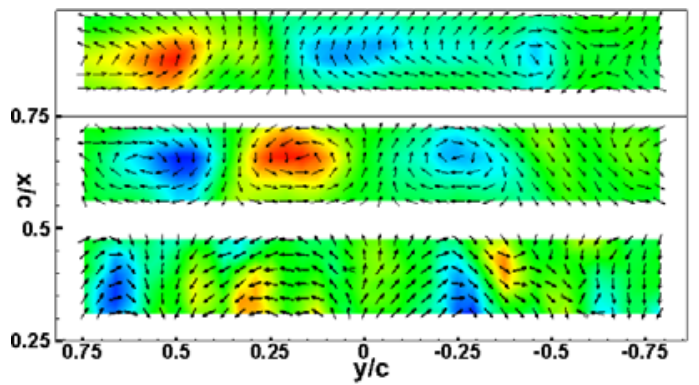

(c)

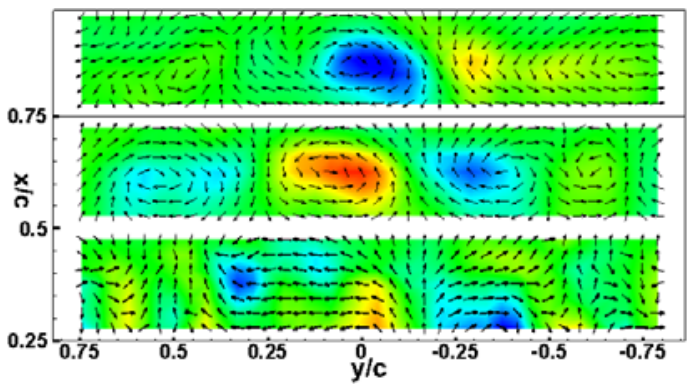

(d)

$8^{\circ}$

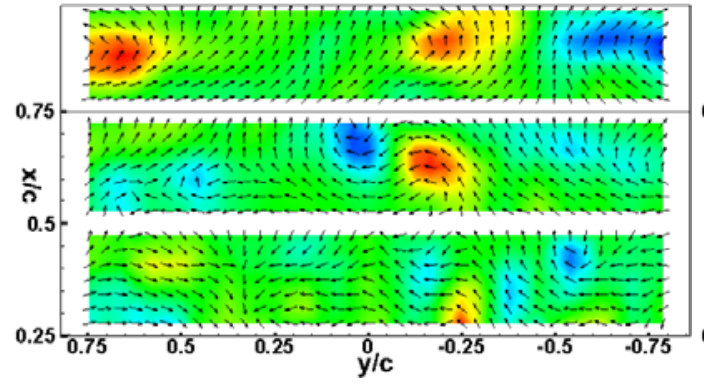

(e)

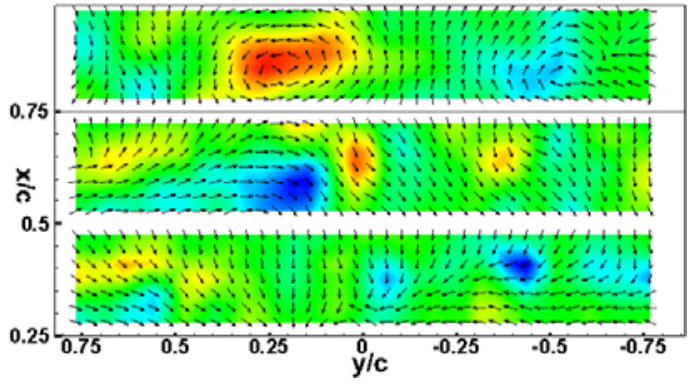

(f)

$12^{\circ}$

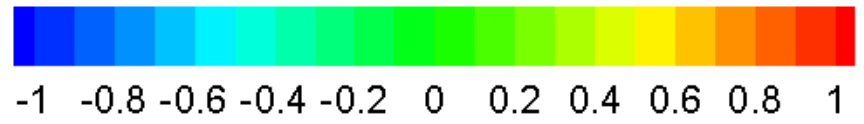

Figure 10. POD mode 1 of $\boldsymbol{\phi}_{\omega_{X}}^{2}$ at Planes $1-3(x / c=0.25,0.5$ and 0.75$)$ with vectors defined by $\phi_{U}^{2}$. Left: $5 \mathrm{CP}$, Right: $9 \mathrm{CP}$ $(\mathrm{a}, \mathrm{b}) 4^{\circ},(\mathrm{c}, \mathrm{d}) 8^{\circ},(\mathrm{e}, \mathrm{f}) 12^{\circ}$

To further investigate isolating the different vortices using the POD modes, the instantaneous snapshots are reconstructed using different number of modes. Figure 11 shows Planes 1-3 colored by the instantaneous $\boldsymbol{\omega}_{\mathbf{z}}$ reconstructed using different numbers of modes (Eq. 8), and isolines defined by the instantaneous $\boldsymbol{\omega}_{\mathbf{z}}$ values calculated from the original instantaneous velocity field. At mode 0, (Figs. 11a-b) the spatial distribution of the vortices approximately represent the 
average values. As mentioned above in the description of Fig. 6, the primary vortices exist in the instantaneous snapshots. These instantaneous primary vortices can be identified by correlating the isolines with the spatial distribution of the primary vortices. This correlation is more obvious at $8^{\circ}$ than at $12^{\circ}$. The clearest matching between the isolines and spatial distribution occurs in the primary vortices at Planes 2 and 3 for the cases at $8^{\circ}$ and $12^{\circ}$, respectively. This is in agreement with the time-averaged results where the largest size and magnitude of the primary vortices occur at these planes. As expected, when using all the modes (Figs. 11c-d), the reconstruction yields the original instantaneous flow field.

When different vortices form by different phenomena, it is possible that different POD modes only capture the corresponding vortices at the particular "energy" level. To distinguish between the vortices in the instantaneous snapshots, the instantaneous flow fields are constructed using the first 10 modes (Figs. 11e-f) and the second 10 modes (Figs. 11g-h). To evaluate the correspondence between the different reconstruction methods and the vortices, the spatial distribution of the reconstructed flow field is correlated with the isolines of the vortices in the original flow field as discussed in Figs. 11a-b. For the case at $8^{\circ}$, the primary vortices and a large number of the vortices in the flow field are captured by the first 10 modes reconstruction (Fig. 11e). However, some smaller vortices are only captured by the reconstruction using the second 10 modes (Fig. $11 \mathrm{~g}$ ) such as the counter rotating vortices in Plane 2 at $y / c= \pm 0.1$ and in Plane 3 at $y / c=-0.55$ and -0.65 . At $12^{\circ}$ the primary vortices (Plane 2 at $x / c=0.3$ and Plane 3 at $x / c=0.25$ in Fig. 11f) and a small number of vortices are captured by the first 10 modes reconstruction. Similar to the case at $8^{\circ}$, using the second 10 modes in the reconstruction yields a better matching of the smaller vortices (Plane 2 at $x / c=0.4$ and 0.5 , and Plane 3 at $x / c=0.5$ in Fig. 11h).

It is concluded from these results that the different size structures in the flow can be captured by reconstructing the flow field using a different number of POD modes. These results also show that primary vortices forming due to the interaction between the backflow and the periodic cambering are the major events in the flow where they are captured in the most energetic modes. However, reconstructing the flow field using only the first 5 modes (not shown) does not yield a good correlation between the reconstructed flow field and the primary vortices. The energy contents in the first and second 10 modes (not including the zero mode) are $42 \%$ and $16 \%$, respectively. This implies that the smaller vortices captured in the reconstruction using the second 10 modes are still major events in the flow and they are not smaller structures similar to those in a turbulent flow. It is likely that these smaller vortices form due to the varying degree of concave down of the periodic cells in the streamwise direction. These smaller structures, however, do not affect the time-averaged results. 


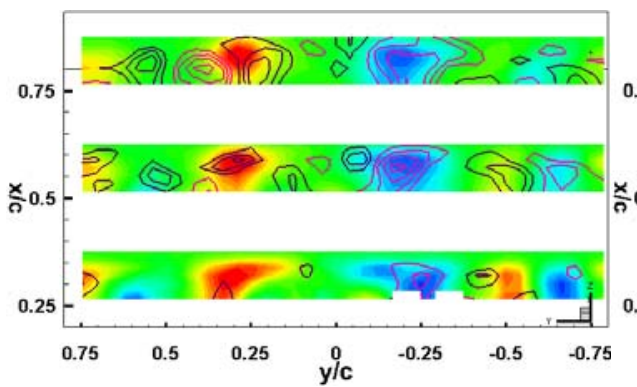

(a)

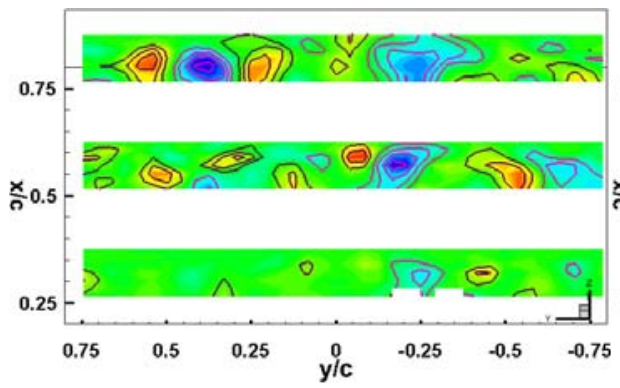

(c)

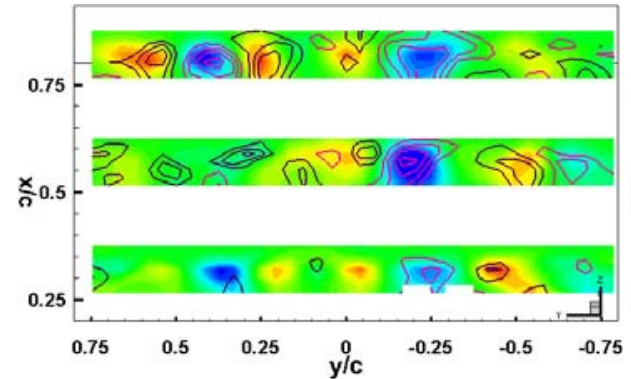

(e)

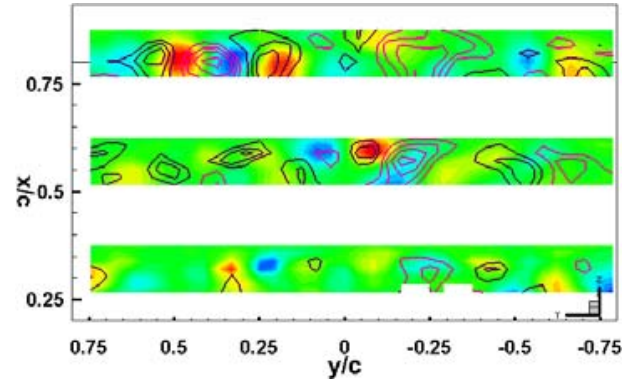

(g)

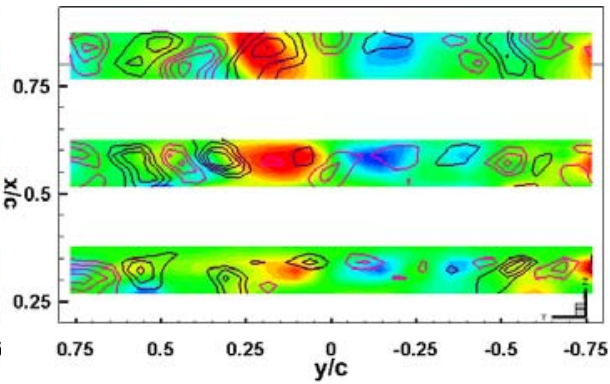

(b)

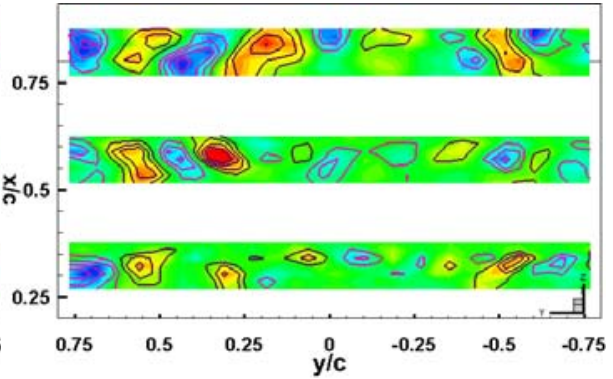

(d)

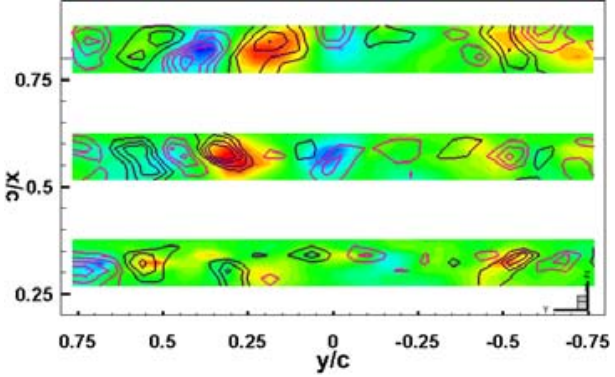

(f)

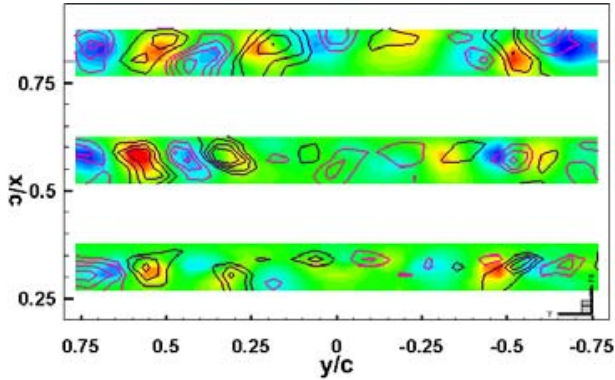

(h)

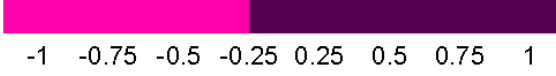

\begin{tabular}{lllllllllll}
\hline 1 & -0.8 & -0.6 & -0.4 & -0.2 & 0 & 0.2 & 0.4 & 0.6 & 0.8 & 1
\end{tabular}

Figure 11. Slices at Planes $1-3(x / c=0.25,0.5$ and 0.75$)$ colored by reconstructed $\boldsymbol{\omega}_{\mathbf{z}}$ using $(\mathrm{a}, \mathrm{b})$ Mode $0(\mathrm{c}, \mathrm{d})$ all modes $(\mathrm{e}, \mathrm{f})$ modes 1-10, $(\mathrm{g}, \mathrm{h})$ modes 11-20. Isolines defined by instantaneous $\boldsymbol{\omega}_{\mathbf{z}}$ Left: $8^{\circ}$, Right: $12^{\circ}$. 


\section{DISCUSSION}

The complex nature of the streamwise and normal primary vortices requires detailed investigation to determine the mechanisms that lead to their formation. In this section, the formation of the vortices is discussed in the context of the streamwise and normal components of the vorticity. The confidence in this approach stems from the fact that the $\lambda_{2}$ values (representing the vortices) correlate well with the vorticity. The two components of the velocity gradient tensor that contribute to the streamwise vorticity are $\frac{\partial \boldsymbol{v}}{\partial \mathbf{z}}$ and $\frac{\partial \boldsymbol{w}}{\partial \boldsymbol{y}}$ (Fig. 12). Both of these components contribute to the primary streamwise vorticity, but the contribution from $\frac{\partial \boldsymbol{v}}{\partial \mathbf{z}}$ is larger. The other gradient, $\frac{\partial \boldsymbol{w}}{\partial \boldsymbol{y}}$, contributes to both the primary and secondary streamwise vorticity. The component, $\frac{\partial \boldsymbol{w}}{\partial \boldsymbol{y}}$, is essentially a product of the component $\frac{\partial \boldsymbol{v}}{\partial \mathbf{z}}$, where it generates due to the interaction of the backflow with the periodically cambered cells.

Consider the following scenario; there is a spanwise flow originating from the wing tip and propagating downstream at a certain angle (angle towards the midspan in the $x-y$ plane). The angle of propagation is set by the strength of the backflow, which becomes larger for higher angles of attack. In a region where the backflow is stronger than the spanwise flow, the fluid flows upstream, whereas the fluid flows in the spanwise direction when the spanwise flow is stronger. Downstream of reattachment, the fluid flows downstream and toward the midspan location (Wahidi and Hubner 2013). The strength of the backflow decreases in the normal direction (i.e., away from the wall) which creates a shift from a purely upstream flow closer to the wall to an oblique downstream flow. This normal gradient of the spanwise flow $\left(\frac{\partial \boldsymbol{v}}{\partial \mathbf{z}}\right)$ contributes to a streamwise vorticity regardless of the presence of the periodic cambering (seen in moderate aspect ratio flat plate at an angle of attack). However, the periodic cambering further contributes to this behavior since both the strength of the reversed flow and the reattachment line vary in the spanwise direction (Wahidi et al. 2014). This is the reason that the streamwise vorticity becomes more organized at $8^{\circ}$ as the backflow region becomes thicker and contains faster upstream flow. As mentioned above, the second contributing factor to the streamwise vorticity is the spanwise gradient of the normal velocity $\frac{\partial \boldsymbol{w}}{\partial \boldsymbol{y}}$. The streamlines are deflected upward near the leading edge and downward downstream of the maximum height location of the separation bubble. When the deflected flow encounters the streamwise vortices that are distributed across the span, it moves downward faster due to the lower pressure in the core of the vortices. This faster downward flow only occurs over the streamwise vortices. This condition creates a spanwise gradient in the normal velocity. Since this spanwise gradient is driven by the low pressure in the streamwise vortices, it has a smaller magnitude and its contribution to the streamwise vorticity is less significant. However, it does create smaller vorticity between the primary vorticity. The smaller vorticity does not have a similar direction of rotation on each side of the plate, but rather has an alternating sign across the span.

The normal vorticity $\left(\omega_{z}\right)$ has two contributing velocity gradients; $\frac{\partial u}{\partial y}$ and $\frac{\partial \boldsymbol{v}}{\partial \boldsymbol{x}}$. As shown in Fig. 13, the excellent agreement between the normal vorticity and $\frac{\partial \boldsymbol{u}}{\partial \boldsymbol{y}}$ is an indication that the spanwise gradient of the streamwise velocity is the major source of this vorticity. This gradient develops due to the difference between the strength of the backflow at the crests and valleys of the periodic cambering (Wahidi et al. 2014), as well as the shape change of the cell in the spanwise direction. This component of the velocity gradient is not confined to the backflow region close to the wall, but exists throughout the viscous region. The streamwise gradient of the spanwise velocity, $\frac{\partial \boldsymbol{v}}{\partial \boldsymbol{x}}$, has a small contribution to the normal vorticity, and is confined to the backflow region.

Another contributing factor to the normal vorticity is the difference in velocities between the crests and valleys of the cells. At some instants of time the flow in the ridges moves in the mean flow direction creating a large difference between the two streams of flow (i.e., backward on the crests and in the streamwise directions in the ridges) that can reach more than 
$0.4 \mathrm{U}_{\infty}$. The difference in velocities creates vortices with an axis perpendicular to the plane of the velocity gradient, hence, normal vorticity. This additional contribution is the reason for the larger magnitude of the normal component of the vorticity and its more organized structure.

The streamwise and normal vorticity form near the leading edge although the cambered cells do not begin until $0.2 \mathrm{c}$ from the leading edge. This means that the cambered cells affect the upstream flow. This occurs when the flow facing the cells accelerates faster whereas the flow facing the valleys accelerates only due to the angle of attack. However, the streamwise and normal components of the vorticity near the leading edge do not present flow circulation as evident by the absence of negative $\lambda_{2}$ values.

The relationship between the streamwise and normal vortices and the behavior of the backflow, which in itself influenced by the periodic cambering, is obvious in the above results. This is the reason for the differences observed in these vortices at different angles of attack. At $4^{\circ}$, the laminar separation bubble covers approximately $0.18 \mathrm{c}$, with smaller thickness and lower backflow velocity (i.e., less negative). These conditions make the interaction between the reversed flow and the spanwise flow less rigorous which results in less organized, smaller and weaker vortices. When the flow is fully separated at $12^{\circ}$, the backflow has much larger magnitude than the spanwise flow, which reduces the oblique flow. Additionally, the larger magnitude of the backflow combined with the larger height of the backflow region decreases the effects of the periodic cambering, thus decreases the magnitude of the vortices. However, the case at $8^{\circ}$ has a backflow region that covers $0.85 \mathrm{c}$ with a balance of backflow velocities and thickness of the backflow region that increases the interaction between the backflow and the spanwise flow, thus increases the effects of the cambered cells.

The interaction of the flow field with the periodic cambering that is based on a time-averaged shape of the flexible membrane wings is seen to produce streamwise and normal vortices. It is speculated that these vortices form on multiplecell flexible membrane wings. The understanding of the behavior and formation of these vortices presented here helps achieving a better understanding and modeling of the flow interaction with multiple-cells flexible wings. 


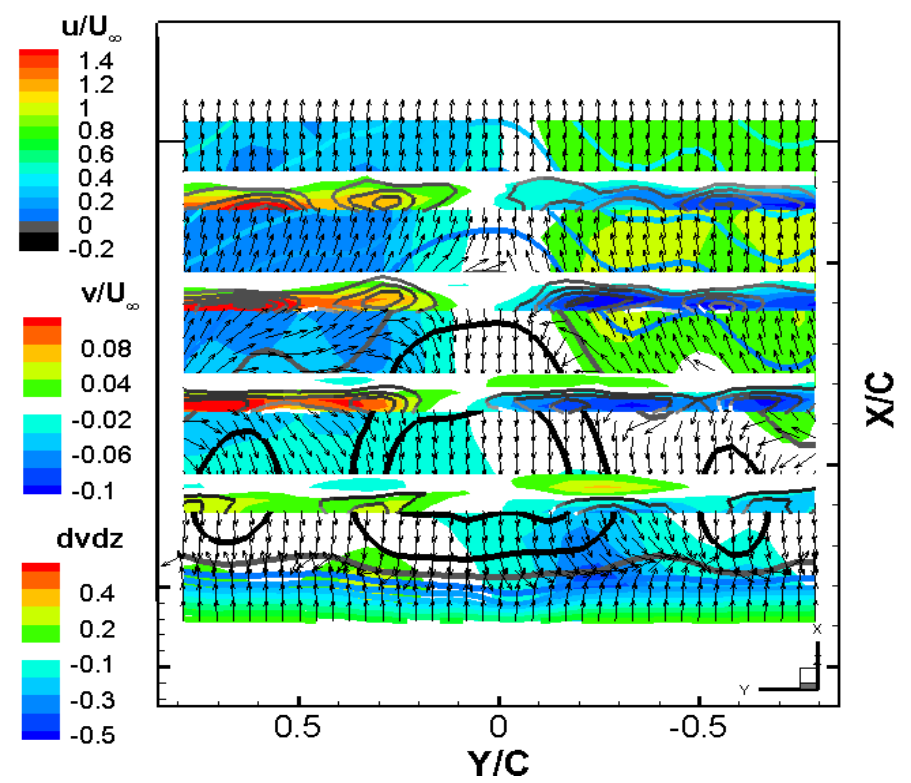

(a)

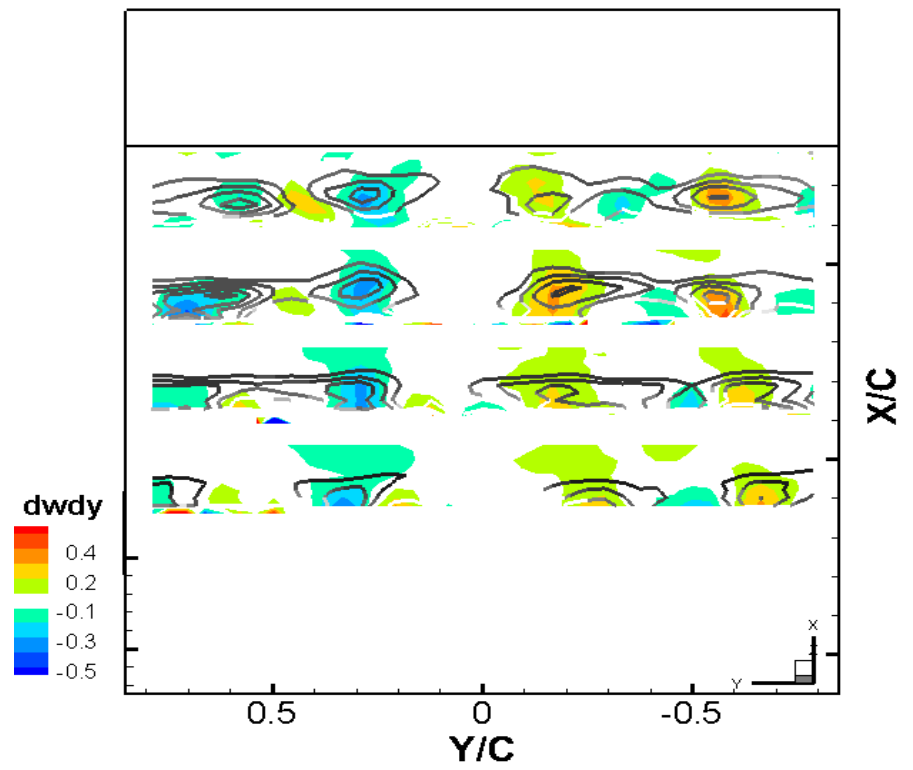

(b)

Figure 12. $y$-z planes at $x / c=0.25-1.0$ colored by (a) $\frac{\partial \boldsymbol{v}}{\partial \mathbf{z}}$ and, (b) $\frac{\partial \boldsymbol{w}}{\partial \boldsymbol{y}}$. Iso-lines defined by $\boldsymbol{\omega}_{\boldsymbol{x}}$ and colored by $\lambda_{2}$. Also shown in (a) $x-y$ plane colored by $\mathrm{v} / \mathrm{U}_{\infty}$ with iso-lines defined and colored by $\mathrm{u} / \mathrm{U}_{\infty} .9 \mathrm{CP}$ at $8^{\circ}$. 


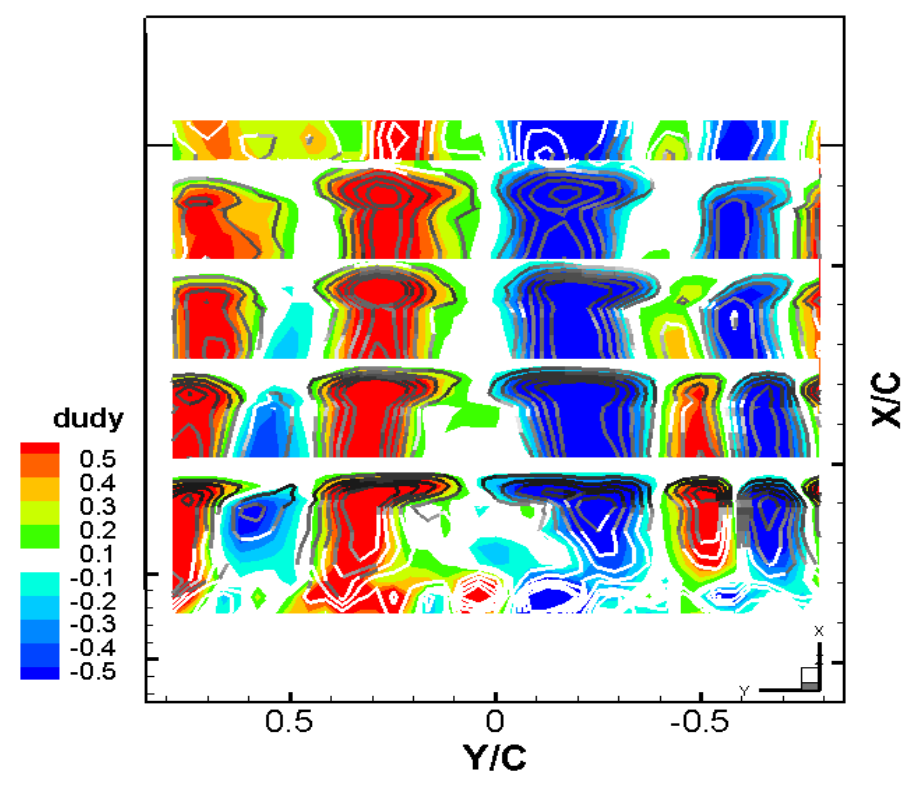

(a)

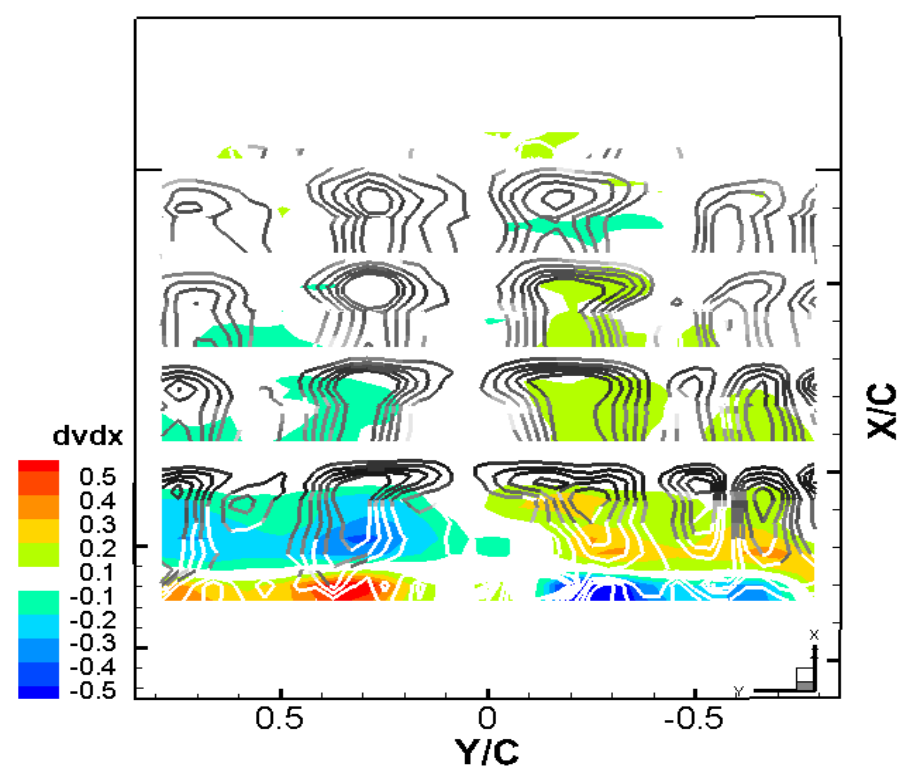

(b)

Figure 13. $y-z$ planes at $x / c=0.25-1.0$ and a $x-y$ plane showing iso-lines defined by $\omega_{z}$ and colored by $\lambda_{2}$ and colored by (a) $\frac{\partial \boldsymbol{u}}{\partial \boldsymbol{y}}$ and, (b) $\frac{\partial \boldsymbol{v}}{\partial \boldsymbol{x}} \cdot 9 \mathrm{CP}$ at $8^{\circ}$. 


\section{CONCLUSION}

The streamwise and normal vortices forming on rigid plates with spanwise periodic cambering are investigated in detail for three different flow fields. These vortices become larger and stronger when a long laminar separation bubble exists in the flow field. The vortices still form for a fully separated flow, but they are less organized. When the flow field is characterized by the presence of a short laminar separation bubble, these vortices are much smaller than the ones in the other cases. The factors that influence the size, magnitude and level of organization are the thickness and strength of the backflow which are functions of the angle of attack. In the long laminar separation bubble case, the backflow is thick and strong enough to create a strong interaction between the spanwise flow induced by the tip vortex and the periodic cells. Smaller streamwise and normal vortices are also observed in the instantaneous snapshots. POD analysis reveals that the primary vortices are captured in the first 10 modes, whereas the smaller vortices are captured in the second 10 most energetic modes. . The ability to isolate the vortices of different sizes using different numbers of modes in the flow field reconstruction suggests that the mechanism of producing the smaller vortices is different than the mechanism producing the primary vortices.

\section{ACKNOWLEDGMENT}

This material is based upon work supported by the National Science Foundation under Grant No. 0958668 and the Air Force Office of Scientific Research (AFOSR) through the grant FA9550-10-1-0152, managed by Dr. Doug Smith. The support of The University of Alabama is also acknowledged through the Research Stimulation Post-Doctoral Fellow Program.

\section{REFERENCES}

[1] Hubner, JP and Hicks, T "Trailing-edge scalloping effect on flat-plate membrane wing performance" Journal of Aerospace Science and Technology 15.8 (2011), pp. 670-680.

[2] Ifju, PG, Albertani, R, Stanford, BK, Claxton, DJ and Sytsma, MJ "Flexible wing micro air vehicles. In: Mueller, T.J. (Ed.), Introduction to the design of fixed-wing micro air vehicles. Including Three Case Studies" AIAA, Reston, VA (2006).

[3] Jeong J. and Hussain, F., “On the identification of a vortex," J Fluid Mech, Vol. 285, 1995, pp. 69-94.

[4] Kostas J, Soria J and Chong MS "A comparison between snapshot POD analysis of PIV velocity and vorticity data" Experiments in Fluids 38 (2005) pp. 146-160.

[5] Lumley J "The structure of inhomogeneous turbulent flows" In: Yaglam AM, Tatrsky VI (eds) Proceedings of the international colloquium on the fine scale structure of the atmosphere and its influence on radio wave propagation, Moscow, Nauka (1967) pp. 166-178.

[6] Sirovich L "Turbulence and the dynamics of coherent structures. Part I: coherent structures" Quarterly Applied Mathematics 45.3 (1987) pp. 561-571.

[7] Tamai, M., Murphy, J. T., and Hu, H., "An Experimental Study of Flexible Membrane Airfoils at Low Reynolds Numbers," 46th AIAA Aerospace Sciences Meeting and Exhibit, AIAA Paper 2008 580, Reno, NV, 7- 10 January 2008.

[8] Timpe, A., Zhang, Z., Hubner, J.P. and Ukeiley, L., "Passive Flow Control by Membrane Wings for Aerodynamic Benefit," Exp Flds, Vol. 54, No. 3, 2013

[9] Wahidi R and Hubner JP "Description of the Vortices and Vorticity around a Low-Aspect Ratio Flat" $51^{\text {st }}$ AIAA Aerospace Sciences Meeting and Exhibit, AIAA Paper 2013-0834, Dallas/Ft. Worth Region, TX, 7- 10 January 2013.

[10] Wahidi, R., Lai, W., Hubner, J. P. and Lang, A., "Time-Averaged and Time-Resolved Volumetric Velocimetry Measurements of a Laminar Separation Bubbles on an Airfoil," European Journal of Mechanics / B Fluids, Vol. 41, 2013, pp. 46-59.

[11] Wahidi R, Hubner JP and Zhang Z "3-D measurements of separated flow over rigid plates with spanwise periodic cambering at low Reynolds number" Journal of Fluids and Structures 49 (2014) pp. 263-282.

[12] Wahidi R, Ölçmen, SM and Hubner JP "POD Analysis of Volumetric PIV Data of Periodically Cambered Plates" 11th International Symposium on Particle Image Velocimetry, Santa Barbara, CA, 14- 16 September 2015. 
[13] Zhang, Z, N Martin, A Wrist, and JP Hubner (2015) "Geometry and Pre-tension Effects on the Aerodynamic Characteristics of Membrane Wings," Journal of Aircraft, DOI:10.2514/1.C033386.

[14] Zhang, Z, L Hopper, A Wrist, JP Hubner and L Ukeiley (2014) "Nondimensional Frequency Scaling of Aerodynamically-Tensioned

Membranes," Journal

of Fluids

and Structures,

48:14-26,

DOI:

2610.1016/j.jfluidstructs.2014.02.005. 